\title{
Dynamic Monitoring of Spatial-temporal Changes in Eco-environment Quality in Beijing Based on Remote Sensing Ecological Index with Google Earth Engine
}

\author{
Jiaqi Lu, ${ }^{1,3}$ Hongliang Guan, ${ }^{1,2}$ Zhiqiang Yang, ${ }^{1}$ and Lei Deng ${ }^{1,2^{*}}$ \\ ${ }^{1}$ College of Resource Environment and Tourism, Capital Normal University, Beijing 100048, China \\ ${ }^{2}$ Engineering Research Center of Spatial Information Technology, Ministry of Education, \\ Capital Normal University, Beijing 100048, China \\ ${ }^{3}$ Beijing Laboratory of Water Resources Security, Capital Normal University, Beijing 100048, China
}

(Received July 28, 2021; accepted September 7, 2021; online published September 21, 2021)

Keywords: Beijing, remote sensing ecological index, eco-environment quality, spatial-temporal changes, Google Earth Engine

The effective and quantitative monitoring of spatial-temporal changes in eco-environment quality is critical for urban sustainable development and policymaking. In this study, four ecological factors (greenness, wetness, heat, and dryness) of Beijing were obtained by integrating MODIS imagery in each year from 2001 to 2020. Then, a remote sensing ecological index (RSEI), which is employed to assess the eco-environment quality, based on the four factors was constructed by using the Google Earth Engine (GEE) platform. Finally, the spatial-temporal changes in RSEI over the 20 years were analyzed. The results show that the eco-environment quality of Beijing improved from 2001 to 2020, with the average RSEI increasing from 0.586 in 2001 to 0.64 in 2020. The eco-environment quality in the mountainous areas of Beijing is higher than that in the urban areas. The area exhibiting deterioration decreased from $23.46 \%$ between 2001 and 2005 to $8.66 \%$ between 2015 and 2020, while the area with improved eco-environment quality increased from $13.04 \%$ between 2001 and 2005 to $21.64 \%$ between 2015 and 2020. We have thus assessed the spatial-temporal changes in eco-environment quality based on RSEI and GEE, which is critical to the investigation of the interactions between human activities and ecosystem services in Beijing.

\section{Introduction}

The ecological impact of urban development is an important area of research and is closely related to human health and the urban eco-environment. In recent years, the industrialization and urbanization of China have been rapid. This change has had a significant impact on the use of land cover and natural landscapes through the growth of built-up areas. ${ }^{(1)}$ However, the rate of urbanization has been more rapid than the self-regulation speed of the ecosystem, resulting in a slew of urban issues such as congestion, waterlogging, the heat island effect, and air pollution. ${ }^{(2)}$

*Corresponding author: e-mail: denglei@cnu.edu.cn https://doi.org/10.18494/SAM.2021.3572 
Therefore, ecological development for big cities is an important problem to consider, and regional planners must prioritize it in a city's construction.

Satellite remote sensing technology is widely used in assessing the quality of the regional eco-environment because it can swiftly, precisely, and thoroughly perform large-scale monitoring. ${ }^{(3,4)}$ Some remote-sensing-based indices have been used to evaluate the health of ecosystems. Xu and coworkers constructed a comprehensive indicator named a remote sensing ecological index (RSEI) involving four indicators (greenness, wetness, dryness, and heat). ${ }^{(5,6)}$ RSEI can reflect the effects of climate change and environmental stresses induced by human activities. ${ }^{(7,8)}$ For example, Yuan et al. applied RSEI in assessing ecological conditions of the Dongting Lake Basin from 2001 to 2019. ${ }^{(9)}$ The results suggested that RSEI can objectively reveal the eco-environment quality on a regional scale. Moreover, using RSEI to assess the ecoenvironment quality can help to prevent variations or mistakes in weight definitions caused by individual characteristics. Yue et al. evaluated the temporal-spatial changes of the ecoenvironment of China's 35 major cities based on RSEI. ${ }^{(10)}$ Firozjaei et al. presented a new method based on RSEI to quantify the urban surface ecological poorness zone (USEPZ) of different cities in Europe. ${ }^{(11)}$ The results showed that RSEI is very useful in modeling the USEPZ intensity (USEPZI) of cities in different environments. Kamara et al. used RSEI to assess the surface water environment in Freetown, Sierra Leone, from 2010 to 2018. ${ }^{(12)}$ The results showed that greenness and wetness originating from surface water make a positive contribution to the eco-environment. However, when RSEI has been used to analyze long-term changes in ecoenvironment quality at a large scale, its construction has been complex and time-consuming, requiring the use of typical specialized remote sensing software such as Environment for Visualizing Images (ENVI).

Google Earth Engine (GEE) is an open-access platform for research. It has been widely used for large-scale studies such as those with global scope. ${ }^{(13-15)}$ Compared with ENVI, GEE is more suitable for RSEI construction and eco-environment quality assessment at a large scale. ${ }^{(16)}$ Therefore, in this study, we used the GEE platform to 1) construct RSEI efficiently by integrating MODIS images and 2) analyze spatial-temporal changes in the eco-environment quality of Beijing from 2001 to 2020. The results will help facilitate appropriate policies in Beijing's ecoenvironment planning and pollution management.

\section{Materials and Methods}

\subsection{Study area}

Beijing $\left(115^{\circ} 42^{\prime}-117^{\circ} 51^{\prime} \mathrm{E}, 39^{\circ} 44^{\prime}-41^{\circ} 06^{\prime} \mathrm{N}\right)$, the capital of China, is situated in northern China. The area has a warm temperate, semi-humid, continental monsoon climate, with four distinctive seasons. The average elevation of the whole city is $43.5 \mathrm{~m}$ and ranges from 20 to 1500 $\mathrm{m}$. The city has 17550000 residents, with a population density of 1069 people per $\mathrm{km}^{2}$. 


\subsection{Data sources}

In this study, the MODIS product collection was used as the data source. The images were derived from NASA Land Processes Distributed Active Archive Center (LPDAAC) Collections (https://lpdaac.usgs.gov/). GEE provides standard data products for various application scenarios, including land surface reflectance, vegetation indicators, climate indicators, and so forth. MODIS data from 2001 to 2020 in a study area can be obtained online using JavaScript API on the platform. Specifically, the greenness component is extracted from the NDVI vegetation index in the MOD13A1 V6 image collection, and the images have a spatial resolution of $500 \mathrm{~m}$. The heat component was obtained from land surface temperature data in the MOD11A2 V6 product, which has a spatial resolution of $1000 \mathrm{~m}$ and gives the average surface temperature over 8 days. Surface reflectance data from the MOD09A1 V6 product were used to build wetness and dryness components. The estimated surface spectral reflectances of MODIS bands $1-7$ are provided in this image set and corrected for atmospheric circumstances (such as gas, aerosol, and Rayleigh scattering).

\subsection{Construction of RSEI}

We selected MODIS images of Beijing to obtain RSEI maps in 2001, 2005, 2010, 2015, and 2020 , spanning 20 years. The acquisition time of these images was from July to September. The four components were all composed at the same time of each year. Because the wetness and dryness components used MODIS reflectance data, we first selected cloud-free images by applying the mask algorithm and cloud pixel detection. Figure 1 shows the workflow of this study.

RSEI, a type of aggregated evaluation index, was specifically developed for assessing the ecological status using remote sensing technology. This index is a function of four indicators (greenness, wetness, dryness, and heat) that are directly related to the ecological quality and may

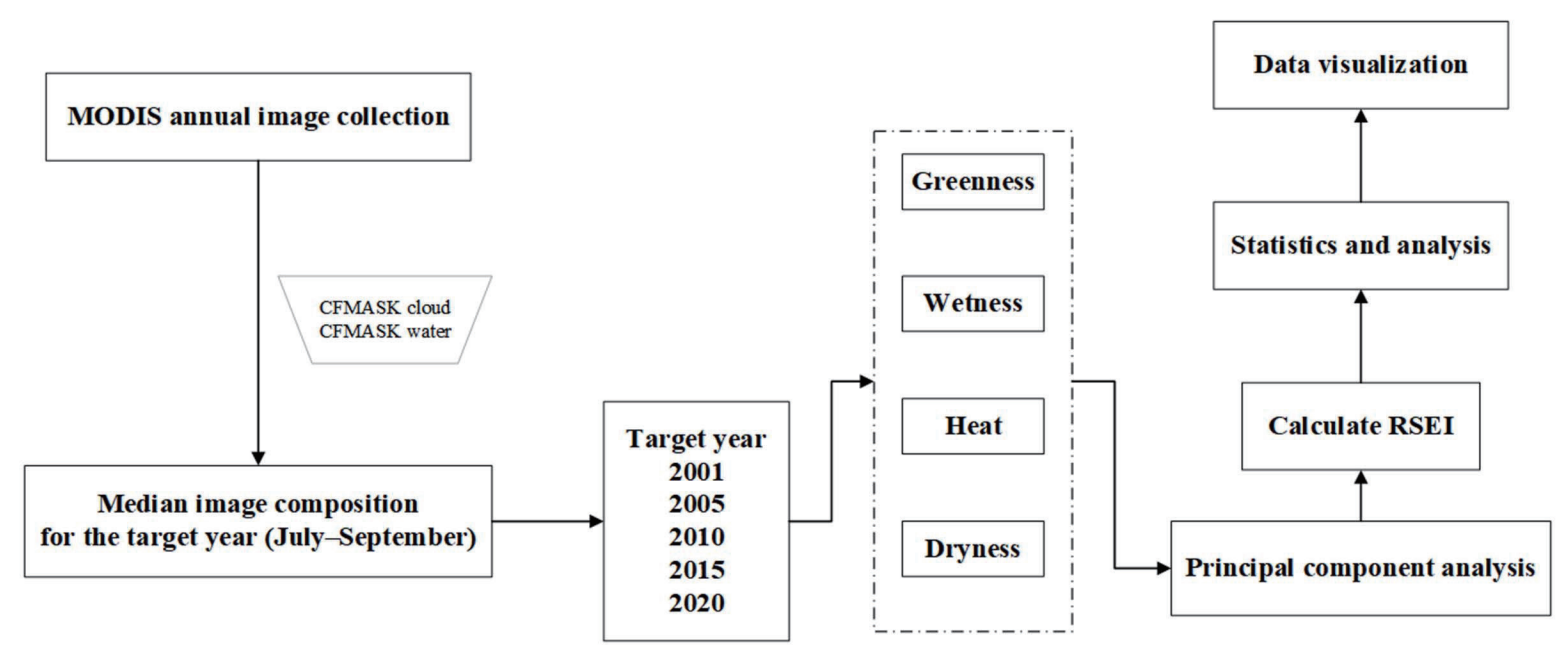

Fig. 1. Workflow of study. 
be sensed by individuals. RSEI was built by using the first component (PC1) of the principal component analysis (PCA) transformation of these four indicators. PCA is a multivariate statistical method used to investigate the correlation between multiple variables. It can effectively recombine many variables with certain correlations into a few bands that retain the information of the initial variables. PC1 usually explains more than $80 \%$ of the total variation of the dataset. The contribution of each indicator to RSEI is weighted by its loading in PC1. This avoids a subjective assignment of the weights of indicators in the weighted sum method. Accordingly, RSEI can be expressed by the following function:

$$
\text { RSEI }=P C 1[f(\text { greenness, wetness, heat, dryness })] \text {. }
$$

(1) Greenness: We used NDVI in MOD13A1 V6 to represent the greenness. NDVI is closely related to plant biomass, leaf area index, and vegetation coverage. Thus, it has long been used as the main ecosystem proxy variable owing to its robustness and simplicity. NDVI is expressed as

$$
N D V I=(\rho N I R-\rho R E D) /(\rho N I R+\rho R E D),
$$

where $\rho R E D$ denotes the red band.

(2) Wetness: The wetness component was obtained from Tasseled Cap transformation. ${ }^{(17)}$ The wetness component reflects the humidity level and is closely related to the quality of the ecoenvironment. Wetness can be expressed as

$$
W e t=0.1147 b_{1}+0.2489 b_{2}+0.3132 b_{3}-0.3122 b_{4}-0.6416 b_{5}-0.5087 b_{6},
$$

where $b_{1}$ denotes the sur_refl_b01 band in MOD09A1 V6.

(3) Heat: We used the daytime land surface temperature (DLST) in MOD11A2 V6 to represent heat. The heat component can directly reflect local climate changes, different types of land use, and the degree of urbanization. In this study, we used DLST directly without modification.

(4) Dryness: We used the normalized difference built-up and soil index (NDBSI) to represent the dryness component. ${ }^{(18)}$ NDBSI reflects the degree of soil desiccation, and it is composed of the index-based built-up index (IBI) and soil index (SI). The calculations are as follows:

$$
\begin{aligned}
& N D B S I=(S I+I B I) / 2, \\
& S I=\frac{[(\rho S W I R 1+\rho R E D)-(\rho B L U E+\rho N I R)]}{[(\rho S W I R 1+\rho R E D)+(\rho B L U E+\rho N I R)]}, \\
& I B I=\left\{\frac{2 \rho \text { SWIR } 1}{\rho \text { SWIR } 1+\rho \text { NIR }}-\left[\frac{\rho \text { NIR }}{\rho \text { NIR }+\rho \text { RED }}+\frac{\rho \text { GREEN }}{\rho \text { GREEN }+\rho \text { SWIR } 1}\right]\right\} /\left\{\frac{2 \rho \text { SWIR } 1}{\rho S W I R 1+\rho \text { NIR }}+\left[\frac{\rho \text { NIR }}{\rho \text { NIR }+\rho \text { RED }}+\frac{\rho \text { GREEN }}{\rho \text { GREEN }+\rho \text { SWIR1 } 1}\right]\right\},
\end{aligned}
$$


where $\rho B L U E, \rho G R E E N, \rho R E D, \rho N I R$, and $\rho S W I R 1$ are the blue, green, red, NIR, and SWIR1 bands of the MODIS image, respectively.

Because the dimensions of the four components are different, we need to normalize their values before performing PCA. In addition, to make horizontal comparisons during the study period, we also need to rescale RSEI to a value between 0 and 1 .

\subsection{Google Earth Engine platform}

GEE is a cloud-based computing platform designed to store and process satellite images and other earth observation data for analysis and decision making. ${ }^{(13)}$ This platform houses a large repository of publicly available geospatial datasets (with data at the petabyte level) in the cloud, including Landsat, MODIS, Sentinel, and other long-term sequence image sets. GEE implements online processing, analysis, and visualization based on the API interface of JavaScript and Python language. ${ }^{(14)}$ The front end is simple to use and provides a suitable environment for interactive data and algorithm development. Users can also add and curate their data and collections, while Google's cloud resources handle all the processing steps.

\section{Results}

\subsection{Overall evaluation of eco-environment quality in Beijing}

Figure 2 shows the changes in the average RSEI of Beijing from 2001 to 2020. The average RSEI over the 20 years is 0.589 , indicating moderate overall eco-environment quality. The ecoenvironment quality gradually declined from 2004 to 2008, then increased from 2008 to 2020. In 2019, RSEI reached a maximum of 0.654 over the 20 years. Overall, the average RSEI of Beijing, and thus the eco-environment quality, improved from 2001 to 2020.

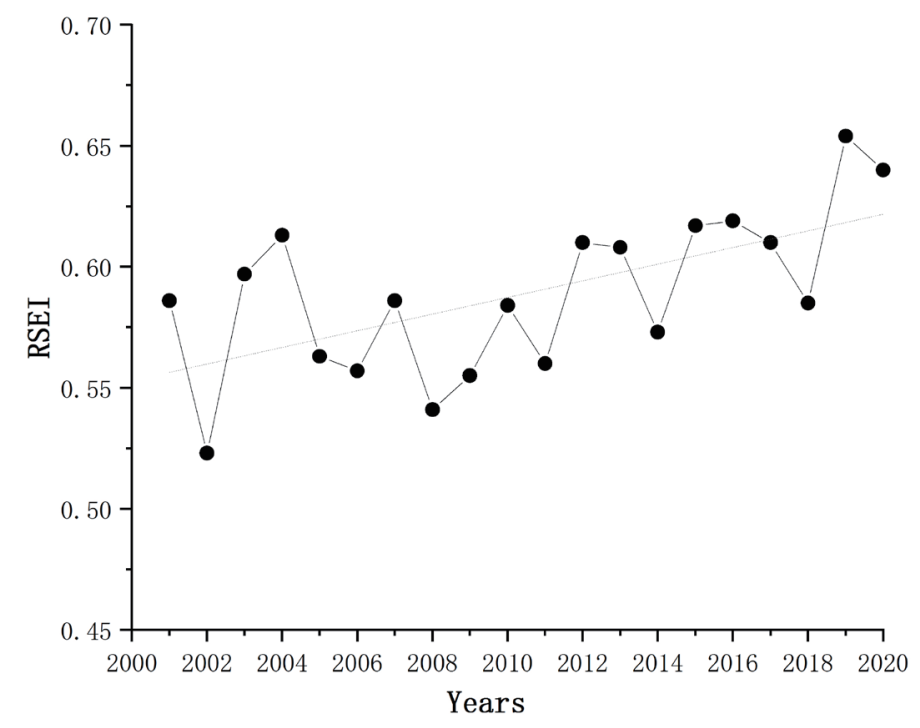

Fig. 2. Average RSEI in Beijing from 2001 to 2020. 
RSEI values closer to 0 represent poor eco-environment quality, while those closer to 1 represent good eco-environment quality, allowing RSEI to be divided into five levels at intervals of 0.2: Level 1: very poor (0-0.2); Level 2: poor (0.2-0.4); Level 3: moderate (0.4-0.6); Level 4: good (0.6-0.8); and Level 5: excellent (0.8-1.0). The area and proportion of each ecological level (very poor, poor, moderate, good, and excellent) were calculated from five RSEI maps of 2001, 2005, 2010, 2015, and 2020. Figure 3 shows the proportion of the area of Beijing with each ecological level over the study period.

The proportion of "very poor" increased from $0.26 \%$ in 2001 to $1.15 \%$ in 2010, then decreased to $0.21 \%$ in 2020 . The most prevalent ecological levels were "moderate" and "good" in 2001, which accounted for 36.68 and $44.08 \%$, respectively, while the other three levels only accounted for $19.24 \%$ of the area of Beijing. The proportions of "moderate" and "good" fluctuated to some extent over the 20 years. The proportion of "excellent" accounted for $20.05 \%$ of the total area in 2020, over 3.2 times that in 2001. Between 2001 and 2015, the overall ecological structure of Beijing did not change significantly, with "moderate" and "good" accounting for more than $70 \%$ of the area. After 2015, with the increase in the proportion of the "excellent" area, the overall ecological structure gradually shifted towards "good" and "excellent". In summary, the RSEI, i.e., ecological level, of Beijing showed a positive trend from 2001 to 2020.

Figure 4 shows the spatial distribution of RSEI levels of Beijing in 2001, 2005, 2010, 2015, and 2020. The overall eco-environment quality was mainly moderate or good. The ecoenvironment quality in the mountainous areas is superior to that in the plain. Beijing's ecological condition deteriorated from 2001 to 2005. Specifically, the areas with good and excellent ecoenvironment qualities were mainly distributed in the northwest and southwest, while those with

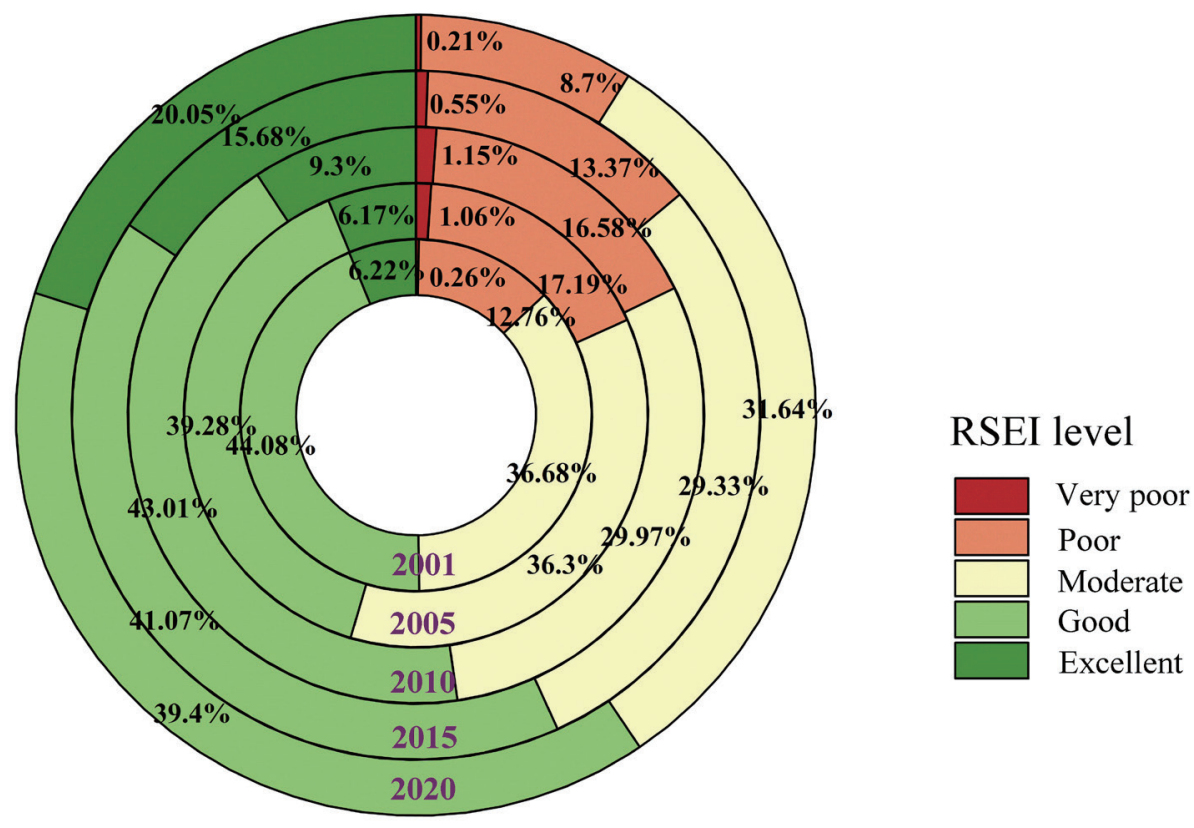

Fig. 3. (Color online) Percentages of the five ecological levels in Beijing from 2001 to 2020. 


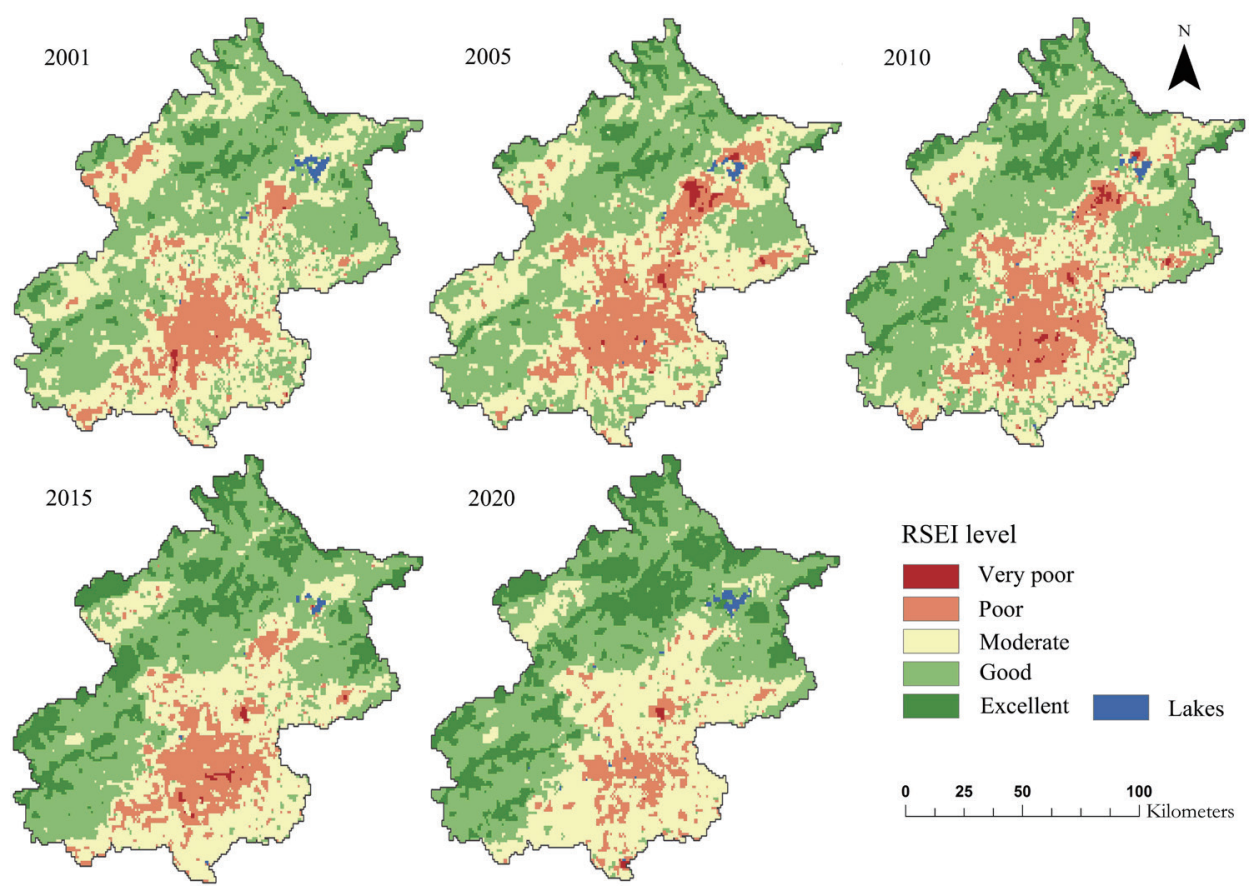

Fig. 4. (Color online) Distribution of RSEI grades in Beijing from 2001 to 2020.

poor and very poor eco-environment qualities were mainly concentrated in urban areas. In 2020, much of the eco-environment of Beijing's mountainous areas reached an excellent level. Compared with 2015, the area with moderate eco-environment quality in the plain was significantly larger in 2020 .

As shown in Fig. 5, RSEI values of 16 districts in Beijing were extracted. To explore the changes in the eco-environment quality of each district, the 16 districts are divided into three categories in accordance with the Beijing urban master plan (2016-2035), which are ecological conservation (Huairou, Yanqing, Mentougou, Miyun, Pinggu, Fangshan, and Changping), central urban (Shijingshan, Haidian, Fengtai, Chaoyang, Dongcheng, and Xicheng), and other areas (Shunyi, Tongzhou, and Daxing). In the last 20 years, the RSEI values of Huairou, Yanqing, and Mentougou, all in the ecological conservation area, have been between 0.6 and 0.8 , indicating good eco-environment quality. The RSEI values of Miyun, Pinggu, Fangshan, and Changping, also in the ecological conservation area, were also greater than 0.5. Except for Pinggu, all districts in the ecological conservation area achieved their highest RSEI in 2020. The RSEI values of Shunyi, Tongzhou, and Daxing have been between 0.4 and 0.6, and the values in 2020 were lower than those in 2001, indicating that the eco-environment quality of these three districts has slightly decreased in the last 20 years. The mean RSEI values of Shijingshan and Haidian, in the central urban area, were between 0.4 and 0.6 during the study period, indicating moderate eco-environment quality, whereas the other districts in the central urban area had lower RSEI values. In 2020, the RSEI values of Fengtai and Chaoyang reached a moderate level. Dongcheng and Xicheng, which are located in the central urban area, had values between 0.2 and 0.4 from 2001 to 2020, indicating poor eco-environment quality. 


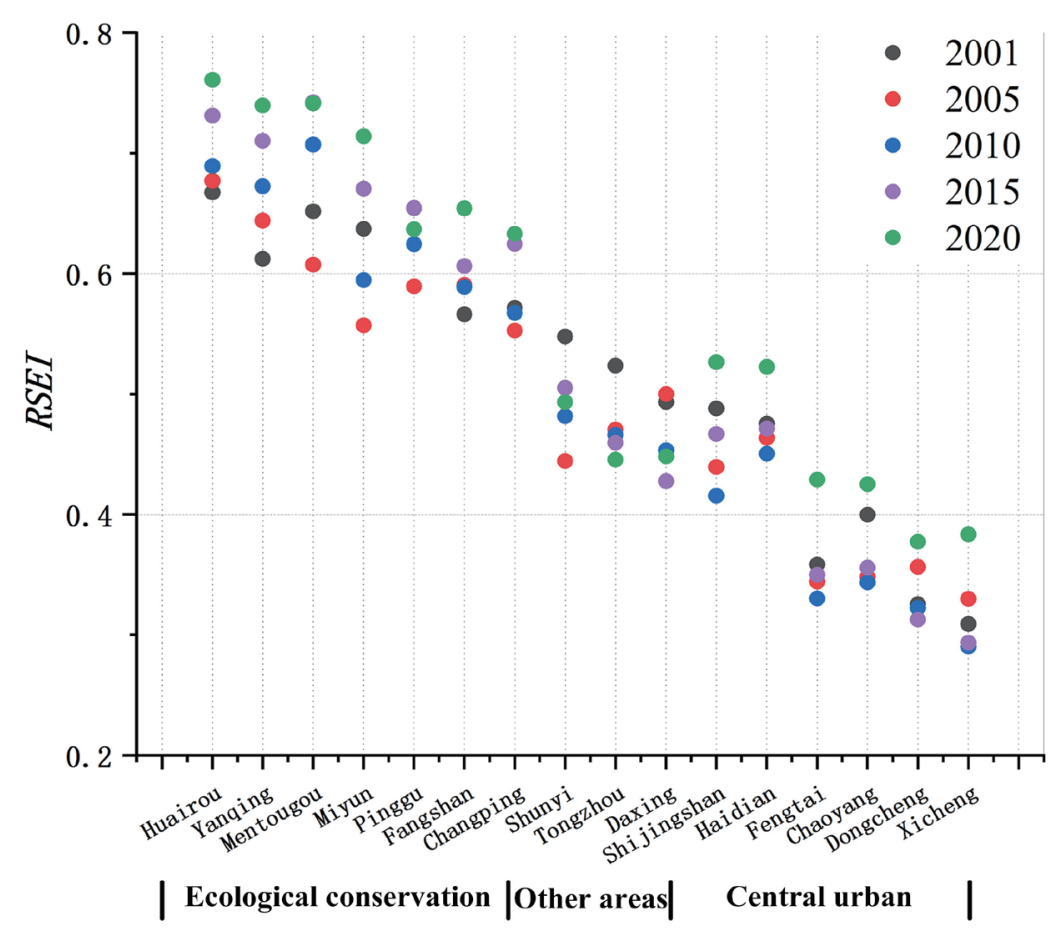

Fig. 5. (Color online) RSEI values of 16 districts in Beijing from 2001 to 2020.

\subsection{Dynamic changes in the eco-environment quality of Beijing}

There is a correlation between the increases and decreases in the areas of different ecological levels in Beijing. The mutual transfer among various ecological levels in different periods is not one-way, and the transferred areas are not quantitative or intuitive. Therefore, to understand the mutual transfer among the different ecological levels, we used a Sankey diagram to analyze the evolution of the areas of Beijing with different eco-environment qualities at intervals of 9 years (2001-2010) and 10 years (2010-2020).

Figure 6 shows that the eco-environment quality of Beijing slightly decreased from 2001 to 2010 but improved from 2010 to 2020. In 2010, the area with a very poor ecological level was $142.9 \mathrm{~km}^{2}$ greater than that in 2001, and the area with a poor ecological level increased by 613.29 $\mathrm{km}^{2}$, with a transfer of $1183.41 \mathrm{~km}^{2}$ from the moderate ecological level. The transferred area from the moderate ecological level to the good ecological level was $1549.60 \mathrm{~km}^{2}$. From 2001 to 2010 , the area with a good ecological level decreased by $165.23 \mathrm{~km}^{2}$ and this change was not very notable. In 2010, the area with the excellent ecological level was $495.69 \mathrm{~km}^{2}$ larger than that in 2001, with an area of $698.88 \mathrm{~km}^{2}$ transferred from the area with a good ecological level. From 2010 to 2020, the area with a very poor ecological level shrunk by $151.1 \mathrm{~km}^{2}$. The transferred area from the poor ecological level to the moderate ecological level was $1639.65 \mathrm{~km}^{2}$. There was little change in the area of the good ecological level, with $1696.96 \mathrm{~km}^{2}$ transferred from the 


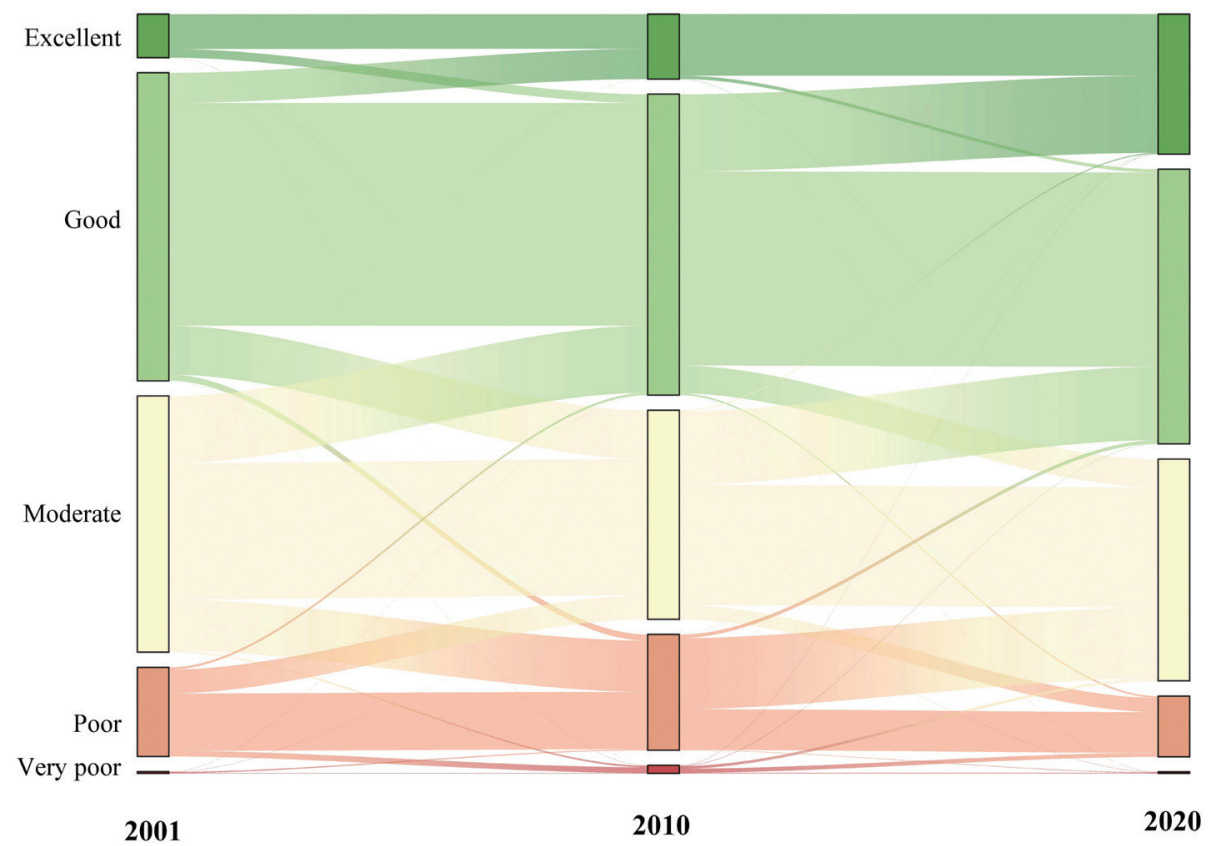

Fig. 6. (Color online) Sankey diagram of the eco-environment quality grade in Beijing from 2001 to 2010 and from 2010 to 2020 .

moderate ecological level. The area with the excellent ecological level greatly increased, owing primarily to the transfer of an area of $1781.81 \mathrm{~km}^{2}$ from the good ecological level. Overall, the ecological condition in Beijing improved from 2010 to 2020.

Table 1 shows the difference in RSEI performance and the spatial-temporal area changes in the eco-environment quality of Beijing based on the results of RSEI level classification in 2001, $2005,2010,2015$, and 2020. The change levels are $+3,+2,+1,0,-1,-2$, and -3 , where, for example, an improvement from "moderate" to "excellent" is a change of +2 . The results show that the eco-environment quality of most regions in the study area remained stable, with no change accounting for $63.50,65.41,65.89$, and $69.70 \%$ in the four time periods. From 2010 to 2020 , the proportion of the negative changes was less than $10 \%$, whereas it was $23.46 \%$ from 2001 to 2005 . The proportion of the positive changes gradually increased, with proportions of $13.04,22.80,25.82$, and $21.64 \%$, and the proportion between 2010 and 2015 was the largest.

Figure 7 shows the spatial-temporal area changes in the eco-environment quality of Beijing based on the difference in RSEI performance. From 2001 to 2005, the area of deterioration was mainly distributed in the east and southwest of Beijing. From 2005 to 2010, the eco-environment quality in most regions remained stable. From 2010 to 2015, the area of deterioration was concentrated in the southern region, and most mountainous areas showed signs of improvement. Between 2015 and 2020, the overall quality of Beijing's eco-environment increased, with only a few regions exhibiting environmental degradation. 
Table 1

Change in RSEI level from 2001 to 2020.

\begin{tabular}{|c|c|c|c|c|c|c|c|c|}
\hline \multirow[t]{2}{*}{ Year } & \multirow[b]{2}{*}{ Change level } & \multicolumn{3}{|c|}{ Improvement } & \multirow{2}{*}{$\begin{array}{c}\text { No change } \\
0\end{array}$} & \multicolumn{3}{|c|}{ Deterioration } \\
\hline & & +3 & +2 & +1 & & -1 & -2 & -3 \\
\hline \multirow{3}{*}{ 2001-2005 } & Area $/ \mathrm{km}^{2}$ & 12.91 & 39.54 & 2052.93 & 10250.50 & 3556.32 & 221.11 & 8.88 \\
\hline & Change area $/ \mathrm{km}^{2}$ & 2105.39 & & & 10250.50 & 3786.30 & & \\
\hline & Percentage/\% & 13.04 & & & 63.50 & 23.46 & & \\
\hline \multirow{4}{*}{ 2005-2010 } & Change level & +3 & +2 & +1 & 0 & -1 & -2 & -3 \\
\hline & Area $/ \mathrm{km}^{2}$ & 0.81 & 99.26 & 3580.53 & 10558.58 & 1853.61 & 47.61 & 1.61 \\
\hline & Change area $/ \mathrm{km}^{2}$ & 3680.59 & & & 10558.58 & 1902.84 & & \\
\hline & Percentage $/ \%$ & 22.80 & & & 65.41 & 11.79 & & \\
\hline \multirow{4}{*}{ 2010-2015 } & Change level & +3 & +2 & +1 & 0 & -1 & -2 & -3 \\
\hline & Area $/ \mathrm{km}^{2}$ & 0.00 & 75.86 & 4005.80 & 10636.77 & 1378.31 & 46.00 & 0.00 \\
\hline & Change area $/ \mathrm{km}^{2}$ & 4081.66 & & & 10636.77 & 1424.30 & & \\
\hline & Percentage $/ \%$ & 25.28 & & & 65.89 & 8.82 & & \\
\hline \multirow{4}{*}{$2015-2020$} & Change level & +3 & +2 & +1 & 0 & -1 & -2 & -3 \\
\hline & Area $/ \mathrm{km}^{2}$ & 0.00 & 46.80 & 3446.57 & 11251.61 & 1380.73 & 16.95 & 0.00 \\
\hline & Change area $/ \mathrm{km}^{2}$ & 3493.37 & & & 11251.61 & 1397.67 & & \\
\hline & Percentage/\% & 21.64 & & & 69.70 & 8.66 & & \\
\hline
\end{tabular}

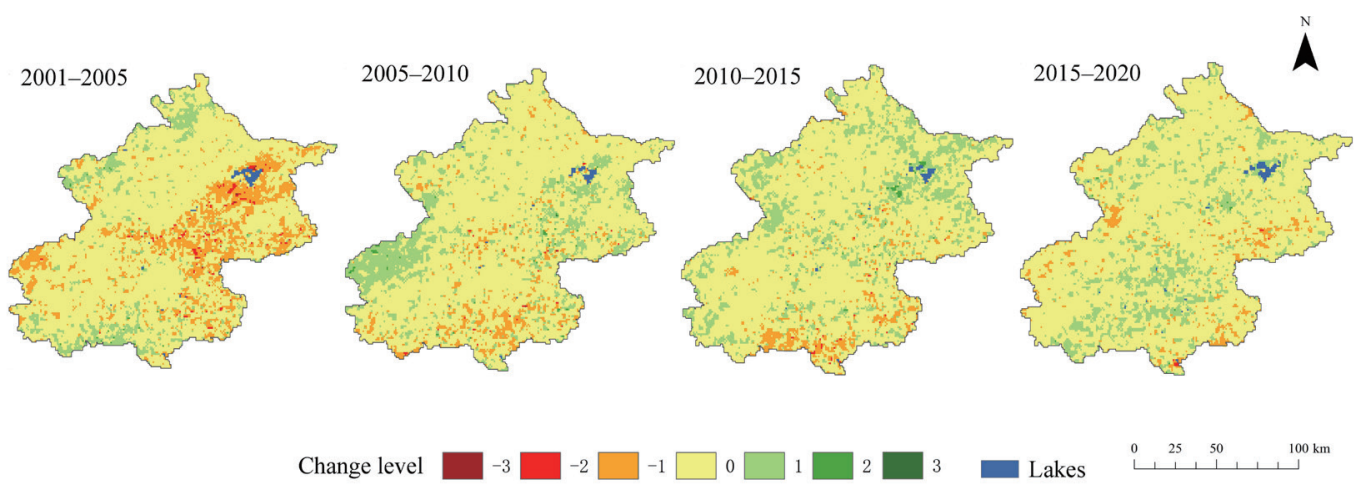

Fig. 7. (Color online) Change in ecological quality in Beijing from 2001 to 2020.

\section{Discussion}

Using the GEE cloud platform, RSEI values of Beijing were calculated to efficiently analyze the regional eco-environment quality. This approach has the following advantages over typical RSEI modeling: (1) It is appropriate for long-term studies. Previously, it was relatively easy to choose suitable images for RSEI calculation when the volume of data was small. Most researchers concentrated on high-resolution satellite images such as Landsat images, which limited the comparability and acquisition time of RSEI. Nowadays, MODIS data provide a large number of images with extremely high time resolution, and it has become very difficult to select data that meet the requirements from the large amount of data. GEE can rapidly process a large 
number of images in a batch and effectively alleviate the challenges faced by RSEI when applied to large areas. Therefore, using the GEE platform and MODIS data to build RSEI can enable the study of long time series. (2) It has the potential for portable monitoring and analysis. GEE provides many built-in codes and functions that are easy to call, such as image synthesis, PCA, and cloud-mask codes. The code used in this study, which was deployed on the GEE platform, was successfully applied to the eco-environment monitoring of Beijing and may easily be adapted for the study of other regions.

Although our method has shown its efficiency for assessing historical spatial-temporal changes in eco-environment quality, some of its limitations will be further examined in future studies. First, RSEI mainly focused on greenness, wetness, heat, and dryness. The ecosystem is a complex and diversified system with many characteristics that are tied not only to natural and social-economic growth but also to the government's organizational and management capacities. Therefore, in future research, we may expand the number of indicators to improve the representativeness of regional ecological quality. Second, GEE's large-scale pixel-level processing of data may encounter some problems such as memory overflow and a low processing speed when the research area is large, which can be improved by image segmentation. In this study, we mainly focused on the use of GEE for long-term eco-environment monitoring in Beijing. However, there was no detailed analysis or discussion of the causes of these changes, which should also be addressed in future research.

\section{Conclusions}

On the basis of the GEE platform and MODIS data, we used RSEI to analyze the spatialtemporal changes in the eco-environment quality of Beijing in the last 20 years. The results show that the average RSEI was within the range of $0.55-0.70$ (0.586 in 2001, 0.563 in 2005, 0.584 in 2010, 0.617 in 2015, and 0.64 in 2020), indicating moderate eco-environment quality. In Beijing, the eco-environment quality in mountainous areas is notably higher than that in plain areas. The proportion of the area with excellent ecological level gradually increased from $6.22 \%$ in 2001 to $20.05 \%$ in 2020 . From 2001 to 2020 , the area with a deterioration of the ecological level decreased from 3786.30 to $1397.67 \mathrm{~km}^{2}$, while the area showing an improvement increased from 2105.39 to $3493.37 \mathrm{~km}^{2}$. The deterioration was concentrated in the eastern region from 2001 to 2005. However, from 2005 to 2020, only a few areas in Beijing showed deterioration.

This research was entirely based on MODIS images, and RSEI was constructed on the GEE platform. We have presented a realistic technique for assessing the spatial-temporal changes in eco-environment quality. More research is needed to connect our methodology with socialeconomic data to investigate the interactions between human activities and ecological services.

\section{Acknowledgments}

This research was supported by Capacity Building for Classified Development - Laboratory Construction - Virtual Simulation Construction Project (grant number 2155165). 


\section{References}

1 Z. Wang, L. Liang, Z. Sun, and X. Wang: J. Environ. Manage. 243 (2019) 227. https://doi.org/10.1016/j. jenvman.2019.04.088

2 Z. Li: Econ. Polit. Stud. 1 (2016) 178. https://doi.org/10.1080/20954816.2013.11673865

3 G. Mateo-García, L. Gómez-Chova, J. Amorós-López, J. Muñoz-Marí, and G. Camps-Valls: Remote Sens. 10 (2018) 1079. https://doi.org/10.3390/rs10071079

4 E. Ivits, M. Cherlet, W. Mehl, and S. Sommer: Ecol. Indic. 9 (2009) 422. https://doi.org/10.1016/j. ecolind.2008.05.013

5 H. Xu, M. Wang, T. Shi, H. Guan, C. Fang, and Z. Lin: Ecol. Indic. 93 (2018) 730. https://doi.org/10.1016/j. ecolind.2018.05.055

6 H. Xu, Y. Wang, H. Guan, T. Shi, and X. Hu: Remote Sens. 11 (2019) 2345. https://doi.org/10.3390/rs11202345

7 D. Zhu, T. Chen, N. Zhen, and R. Niu: Environ. Sci. Pollut. Res. 27 (2020) 15716. https://doi.org/10.1007/ s11356-020-08054-2

8 Y. Xiong, W. Xu, N. Lu, S. Huang, C. Wu, L. Wang, F. Dai, and W. Kou: Ecol. Indic. 125 (2021) 107518. https:// doi.org/10.1016/j.ecolind.2021.107518

9 B. Yuan, L. Fu, Y. Zou, S. Zhang, X. Chen, F. Li, Z. Deng, and Y. Xie: J. Cleaner Prod. 302 (2021) 126995. https://doi.org/10.1016/j.jclepro.2021.126995

10 H. Yue, Y. Liu, Y. Li, and Y. Lu: IEEE Access 7 (2019) 51295. https://doi.org/10.1109/access.2019.2911627

11 M. K. Firozjaei, M. Kiavarz, M. Homaee, J. J. Arsanjani, and S. K. Alavipanah: Sci. Total Environ. 757 (2021) 143755. https://doi.org/10.1016/j.scitotenv.2020.143755

12 D. M. Kamara, Z. Yang, and Y. A. J. G. Lahai: GSJ 8 (2020) 323. https://doi.org/10.11216/gsj.2020.12.45978

13 J. Xiong, P. S. Thenkabail, M. K. Gumma, P. Teluguntla, J. Poehnelt, R. G. Congalton, K. Yadav, and D. Thau: ISPRS J. Photogramm. Remote Sens. 126 (2017) 225. https://doi.org/10.1016/j.isprsjprs.2017.01.019

14 H. Tamiminia, B. Salehi, M. Mahdianpari, L. Quackenbush, S. Adeli, and B. Brisco: ISPRS J. Photogramm. Remote Sens. 164 (2020) 152. https://doi.org/10.1016/j.isprsjprs.2020.04.001

15 O. Mutanga and L. Kumar: Remote Sens. 11 (2019) 591. https://doi.org/10.3390/rs11050591

16 R. Kennedy, Z. Yang, N. Gorelick, J. Braaten, L. Cavalcante, W. Cohen, and S. Healey: Remote Sens. 10 (2018) 691. https://doi.org/10.3390/rs10050691

17 M. H. A. Baig, L. Zhang, T. Shuai, and Q. Tong: Remote Sens. Lett. 5 (2014) 423. https://doi.org/10.1080/21507 $04 x .2014 .915434$

18 X. Hu and H. Xu: Ecol. Indic. 89 (2018) 11. https://doi.org/10.1016/j.ecolind.2018.02.006 